\title{
The effect of flanking context on visual classification: The joint contribution of interactions at different processing levels
}

\author{
JOHN H. FLOWERS and NOREEN WILCOX \\ University of Nebraska, Lincoln, Nebraska
}

\begin{abstract}
Flanking characters that surround a target character may cause either facilitation of or interference with target classification, depending on experimental context. In three different experiments, the patterns of facilitative priming and interference were shown to change systematically as a function of onset asynchrony between flankers and target, illustrating differing time courses of the overlapping processes that each contributes to overall reaction time performance.
\end{abstract}

The present series of experiments was concerned with the general question of how responding to a target in a visual display is affected by the presence of other visual events that surround it in space or time. Related experiments have often been described by terms such as the "effect of visual noise" on target discrimination (e.g., Bjork \& Murray, 1977; Eriksen \& Hoffman, 1973) and have emphasized disruptive or interference effects of spatially adjacent elements of the display on either speed or accuracy of target classification. However, in cases in which the onset of the flanking noise elements precedes the onset of the target, such a task may take on characteristics of a "forward masking" experiment or perhaps even a "semantic priming" experiment. In such cases, the elements that surround the target may produce facilitative as well as disruptive effects (Eriksen \& Schultz, 1979; Flowers, 1980; Taylor, 1977). Since it is clear that interactions between the noise and target may occur at a variety of levels or stages, even within a single experimental task, the systematic manipulation of the stimulus onset asynchrony (SOA), relative to manipulation of the noise or context, represents one potentially useful approach for isolating interactions occurring at different stages (Taylor, 1977).

\section{Perceptual- vs. Encoding- and Response-Level Interactions}

An examination of previous experimental evidence on what occurs when human observers attempt to de-

This research was supported by NSF Grants IST-7911162 and IST-811054 to John H. Flowers. The authors would like to thank Jane Loseke for assistance in data collection. Portions of this research were presented at the 22nd annual meeting of the Psychonomic Society, Philadelphia, Pennsylvania, November 1981. The authors' mailing address is: Department of Psychology, University of Nebraska, Lincoln, Nebraska 68588. tect, identify, or classify a target character embedded among surrounding characters reveals many findings in common (e.g., interference effects decrease as a function of the spatial separation between the target and flankers). However, some interesting discrepancies also exist. One of the most striking discrepancies concerns the effect of flankers that are visually similar or are identical to the target.Those researchers who have emphasized perceptual level interactions cite evidence showing that performance tends to be worse when the flankers and target elements are identical than when they are visually different. In addition, Egeth and Santee (1981) have shown that flankers sharing the same name as the target, but which are visually different (upper- vs. lowercase letters), tend to interfere with target recognition to a greater extent than do flankers that have names different from those of the target. On the other hand, a variety of other experiments (e.g., Eriksen \& Eriksen, 1974; Eriksen \& Hoffman, 1973; Eriksen \& Schultz, 1979; Taylor, 1977) have all tended to support a decrement in interference effects, if not actual facilitation of target classification speed, when the flankers and the target are identical or are associated with the same response category. Thus, evidence for both increased interference and facilitative priming can be found in situations in which subjects are required to recognize or respond to a target character surrounded by other identical characters.

Santee and Egeth (Note 1) have pointed out that interference effects that are accentuated by target-noise similarity are typical of tasks in which accuracy is the dependent variable and for which performance is constrained by brief stimulus exposure and masking, whereas facilitative priming is more typical of reaction time tasks involving stimuli of high visibility. Although such methodological distinctions may change the relative contributions of perceptual-processing limitations vs. limitations occurring at "higher" levels (such as response genera- 
tion) to overall performance, it still remains possible that the "earlier" perceptual interactions may influence performance in tasks involving highly visible unmasked stimuli. ${ }^{1}$ For example, potential response-priming benefits may be attenuated or eliminated by perceptual grouping phenomena, feature competition, etc., that occur when flankers are physically identical to the target. Specific evidence that perceptual interactions may compete with encoding or response-generation effects can be found in experiments reported by LaBerge (1981) in which flankers identical to the target produced much slower reaction times (RTs) than did flankers that were physically different but were associated with the same response as the target. If this study had manipulated SOA instead of exclusively using displays in which the targets and flankers appeared simultaneously, it seems possible that facilitative priming might have been obtained even in the identical-flanker conditions, provided that the flankers preceded the onset of the target. "Perceptual" interactions, including such peripheral phenomena as contour masking, feature extraction competition, and grouping/localization processes, may be limited to cases in which the target and flankers occur simultaneously and might diminish substantially with temporal separation between flankers and targets.

\section{Response Priming vs. Other Categorization Processes}

Just as one can theoretically distinguish "early" perceptual interactions between a visual target and surrounding display elements from interactions occurring at higher levels, it is possible to differentiate between different forms of "higher level" interactions-for example, those involving direct response competition vs. those involving categorization or "internal recognition responses" (Eriksen \& Eriksen, 1974; LaBerge, 1981). A common theme throughout studies of the Stroop effect, for example, has been whether color-word interference is an "encoding" or a "response selection" process (Duncan-Johnson \& Kopell, 1981; Hock \& Egeth, 1970). While the recent criticisms of discrete linear stage models of visual information processing raise questions about the usefulness of attempting to isolate interactions within a single process via experimental manipulations such as additive factors (e.g., Eriksen \& Schultz, 1979; McClelland, 1979; Miller, 1982), systematic SOA manipulations provide a possible approach for separating interactions that occur in stages that may partially overlap in time (Taylor, 1977).

Taylor (1977) has shown how systematic manipulation of SOA in a flanking-context letter-classification task can illustrate different time courses of involuntary priming effects (when the flanking context makes no probabilistic prediction about the target) and responsebiasing effects (when the subject knows that a particular flanker predicts the response on $80 \%$ of the trials). Taylor's study also suggests that SOA manipulation can be highly useful in separating different classes of in- voluntary effects as well. Of particular interest to our present study is Taylor's finding (1977, Experiment 3) that involuntary facilitative priming of target classification seems to be limited to displays in which the target letter and priming letters are identical; when the target letter was surrounded by a different letter associated with the same response button, RTs were nearly identical to those obtained when the target was surrounded by a "neutral" letter associated with neither response category.

Other data, however, have suggested that facilitative priming may not be limited to simple stimulus repetition. Eriksen and Eriksen (1974) have clearly shown response-mapping effects on $\mathrm{RT}$ as well as a repetition effect per se, although their results were largely interpreted in terms of differing amounts of interference as opposed to facilitation. In addition, LaBerge (1981) and Shaffer and LaBerge (1979), who did not vary SOA, have shown effects on choice RT of both the response compatibility of a flanker with its target and its semanticcategory compatibility. Mixtures of letters and digits or mixtures of words belonging to different semantic categories (e.g., IRON CHAIR IRON) produced longer classification times than did targets and flankers belong. ing to the same semantic category, even when the flankers and target were mapped to the same response key. It thus seems possible, given the appropriate combination of stimulus alternatives in the experiment, to obtain both response-priming effects and semanticcategory-priming effects. These different types of involuntary priming effects may occur at different processing levels, and thus a systematic manipulation of SOA might provide a means of assessing their degree of interdependence and their relative time course.

\section{Do Facilitative and Inhibitory Priming Effects Involve Different Processing Levels?}

There is some evidence that the relative amount of RT facilitation by "compatible" flanking characters as compared with interference from "incompatible" flankers may depend upon stimulus and task variables in addition to those involving the perceptual-level interactions among identical stimulus elements discussed earlier. Taylor (1977) has suggested that increases in the number of stimulus alternatives (stimulus uncertainty) lead to a greater degree of facilitative priming, whereas interference effects are more dominant with very simple response mappings. Some data from our laboratory (Flowers, 1980) has suggested that the spatial separation between the flankers and the target may affect the relative amount of facilitation and interference in a similar manner, with facilitation being evident at spatial separations that largely eliminate interference effects.

Any stimulus or task manipulation that differentially affects the relative amount of facilitative vs. inhibitory priming would suggest that compatible and incompatible flankers may interact with target processing at different 
levels. On the basis of his data, Taylor (1977) suggested that facilitative priming might be related to resolving difficulty associated with response selection, while interference effects involve disruption of earlier encoding processes. Although it is not obvious that this interpretation can account for effects of target-flanker spacing, a systematic manipulation of a number of variables including spacing and stimulus uncertainty may shed additional light on differences between facilitative and disruptive priming effects.

Unfortunately, a major problem in quantifying interference effects and facilitation effects is the selection of the baseline condition. While this problem, which is common to other types of priming tasks, such as lexical decision (e.g., Neely, 1977; Schvaneveldt \& Meyer, 1973), may have no "ideal" solution, the fact that different investigators have used different approaches makes it very difficult to make comparisons between experiments. One of the major goals throughout the present series of experiments was the documentation of interference and facilitation effects as a function of SOA, using a relatively common display procedure and a nonalphanumeric character (pound sign or asterisk) as the neutral baseline condition. Thus, as in the study conducted by Taylor (1977), changes in the RT functions across SOA levels resulting from various stimulus and task manipulations can be observed and associated with presumed underlying processing stages.

\section{EXPERIMENT 1}

Experiment 1 was conducted to examine the changing patterns of interference and facilitation of digit naming caused by "compatible" and "incompatible" flanking digits as a function of SOA and the spatial separation between the target digit and the flankers. This experiment was essentially a replication of an experiment reported by Flowers (1980), but with two changes in procedure. First, a "neutral" flanker, a pound sign (\#), was intermixed in blocks of trials at each SOA level, along with the response-compatible and responseincompatible flanker-target combinations. Second, while the experiment conducted by Flowers (1980) included an equal proportion of compatible and incompatible flankers within a block of trials, the stimulus-generation procedure led to a correlation between the flanker and target digits such that the single "best guess" of the target given the flanker $(p=.5)$ was the digit identical to the flanker. In Experiment 1, completely uncorrelated pairings of flanker and target digits were employed to eliminate the possibility of any anticipatory guessing strategy. Speeding or slowing of target naming relative to the neutral (\#) flanker condition could thus be attributed to the sort of "automatic" activation effects found in other character-classification experiments similar to those of Eriksen and his colleagues and to that of Taylor (1977).

\section{Method}

Subjects. Eight students at the University of Nebraska received $\$ 5.00$ for participating in two $1-h$ experimental sessions. All had normal or corrected vision, and all spoke English as their native language.

Apparatus. An Automated Data Systems 1800 A computer was used to control the stimulus display, to time the subjects' responses, and to store response latencies. The stimuli were displayed on a Tektronix Model 604 display scope employing a P31 phosphor. A Lafayette voice relay served as the response device. The subject used a telegraph key to initiate each trial. The display scope, voice relay, and telegraph key were all located on a table at which the subject was seated. No device was used to maintain a fixed viewing distance, but, given the position of the chair, the distance between the scope and the subjects' eyes averaged approximately $45 \mathrm{~cm}$.

Conditions. The independent variables were the flanker condition (incongruent, congruent, and neutral), the SOA between flanker and target $(0,50,100,200$, or $500 \mathrm{msec})$, and the spatial separation (narrow, $1.1 \mathrm{deg}$ of separation between the target and flankers; wide, $3.3 \mathrm{deg}$ of separation between the target and flankers).

In each experimental session, half of the subjects were presented first with 10 different blocks of trials under the narrow-separation condition and then with 5 blocks under the wide-separation condition. This order was reversed for the second experimental session. The remaining subjects received the complementary order of trials.

Each group of five blocks, during which the target-flanker separation was held constant, consisted of one block for each of the five SOA values. The first three trials in each block were practice trials and were omitted from the data analysis. The remaining trials consisted of 24 congruent displays (e.g., 222, 555 , etc.), 36 incongruent displays (e.g., 454, 323, etc.), and 12 neutral displays (e.g., \#4\#, \#3\#, etc.). The digits $2,3,4$, and 5 appeared 18 times in each block. Thus, the number of displays was not balanced across flanker type (congruent, incongruent, and neutral) but was instead balanced across combinations of flankers and target. Thus, whenever a flanker appeared, there was an equal probability that the target would be a 2 , a 3 , a 4 , or a 5 . The order of the displays within each block and the order of the blocks were determined by a pseudorandom process for each subject and for each session.

Procedure. Before an experimental session, each of the subjects was seated at the table for about $5 \mathrm{~min}$ in the dimly lit experimental room, to allow for dark adaptation. The subjects initiated each of the 75 trials in each block by pressing a telegraph key. Depressing the key signaled the onset of a fixation field consisting of a horizontal row of three zeros centered on the screen. These zeros marked the positions in which the stimulus display (the target and two horizontally flanking characters) were to appear. At the assumed viewing distance of $45 \mathrm{~cm}$, each of these zeros subtended a vertical visual angle of approximately $.9 \mathrm{deg}$. The flanking zeros were replaced by the flanking context digits $800 \mathrm{msec}$ after the appearance of the fixation row. The center zero was then replaced by the target digit after an SOA delay of $0-500 \mathrm{msec}$.

The subjects responded by vocalizing the name of the target digit "as rapidly as possible, avoiding errors." The triggering of the voice relay terminated the screen display and instructed the computer to store the response latency. The experimenter followed the subjects' responses with an answer key and marked any trials in which an error, stuttering, or premature vocalization occurred. Latencies from such trials (only $1.6 \%$ of all trials) were not included in the data analysis.

\section{Results and Discussion}

Given the extremely low error rates (1.6\%), reaction times were used exclusively as the dependent variable 
in the data analysis from this experiment. ${ }^{2}$ Figure 1 displays the mean reaction times, averaged across subjects, for each grouping of SOA and target-flanker combination. The top panel summarizes the data from the narrow displays (separation of $1.1 \mathrm{deg}$ of visual angle), and the bottom panel presents the data obtained using the wide ( $3.3 \mathrm{deg}$ of visual angle) displays.

In Figure 1 (top), it can be seen that there is a clear separation between the RT values for the three compatibility levels of the flanker and that these values are ordered in the expected direction (identical < neutral $<$ incompatible). This main effect of flanker compatibility was statistically significant $[F(2,14)=21.3$, $\mathrm{p}<.01]$. However, as Figure 1 suggests, the size of the compatibility effect did not remain constant across SOA levels; a significant interaction between SOA and compatibility also occurred $[\mathrm{F}(8,56)=7.5, \mathrm{p}<.01]$. In addition to the attenuation of the compatibility effect at the 200-msec SOA level, Figure 1 (top) suggests a buildup of the effect during the first $100 \mathrm{msec}$, a buildup generally comparable to that shown by Flowers (1980). Evidence of facilitation (faster RTs to the compatible than to the neutral flanker stimuli) occurred only when the flanker led the target. This finding raises the possibility that facilitative priming brought about by flankers identical to the target has to "overcome" some

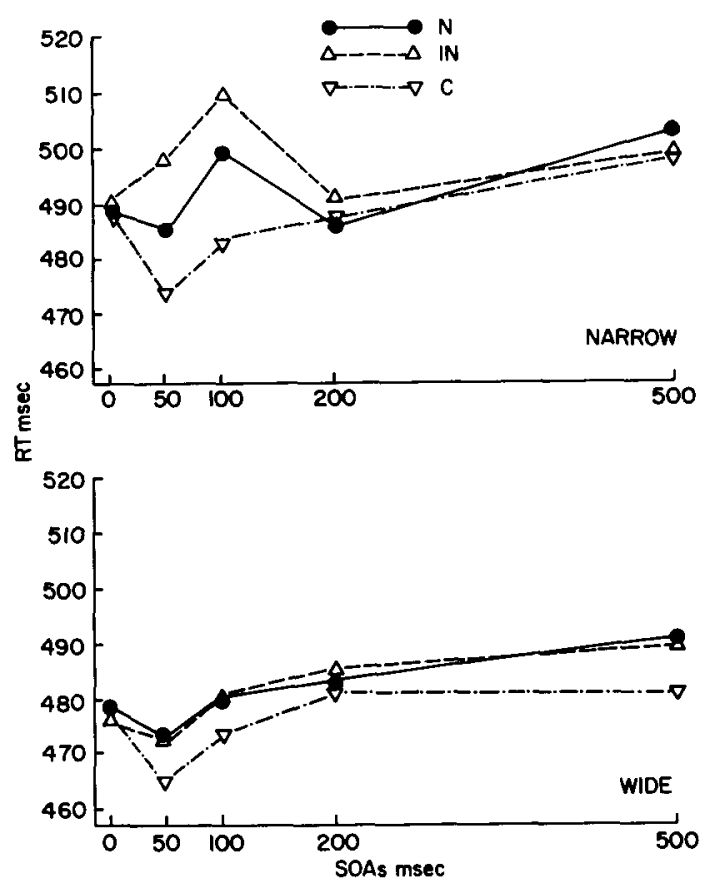

Figure 1. Voice RTs from Experiment 1 plotted as a function of flanker condition and SOA. The top panel presents data obtained with the narrow $(1.1-\mathrm{deg})$ target-flanker separation: the bottom panel presents data obtained with the wider (3.3-deg) separation. In Figures 1-3, downward-pointing open triangles indicate response-compatible flanker conditions, upward-pointing open triangles indicate response-incompatible flanker conditions, and solid circles indicate conditions involving a neutral or baseline flanker. initial perceptual interference that occurs when very similar visual features are presented simultaneously.

Interference effects, as measured by slower RTs to the incompatible than to the neutral distractor displays, are evident for SOAs of $200 \mathrm{msec}$ and less-a pattern generally similar to that found by Taylor (1977). It should be pointed out, however, that the relatively small difference of $3.1 \mathrm{msec}$ between the incompatible and neutral conditions at the $0-m s e c$ SOA is anomalous in comparison with the $10-20-\mathrm{msec}$ or greater differences found for comparable conditions in experiments by other investigators (e.g., Taylor, 1977) and in other experiments from our laboratory that used similar equipment and procedures (e.g., Flowers, 1980, Note 2). Furthermore, the following experiments, which involved small distances between targets and flankers, typically produced considerably larger interference effects at 0 -msec SOA. ${ }^{3}$

With the wider separation between the flanker and target (Figure 1, bottom), the size of the flankercompatibility effect was substantially reduced, although a significant main effect was still noted $[F(2,14)=6.9$, $\mathrm{p}<.01]$. A visual comparison of the top and bottom panels shows that, across SOA levels, the pattern of the effect of wide-separation flanker conditions differed substantially from the pattern obtained when the flankers were adjacent to the target. First, with the wide separation, it is apparent that virtually all of the flanker "effect" results from faster RTs to the compatible flanker trials, compared with the other conditions. RTs for the incompatible-flanker trials were actually slightly faster than for the neutral-flanker trials, except at the 200-msec delay interval. Thus, the effect of increasing the spatial separation between the flanker and the target digit might be described as eliminating the interference effects of incompatible noise while still maintaining some benefit from facilitative priming by compatible noise. Second, as opposed to the pattern of results obtained with the narrow displays, the size of the facilitation effect remained relatively constant across SOA levels; no interaction between SOA and flanker type was revealed statistically $[F(8,56)=.89]$.

Combining data from both spatial separations into a single ANOVA thus revealed a significant triple interaction between spacing, flanker conditions, and SOA $[F(8,56)=2.79, p<.05]$. A significant three-way interaction between these same variables was also obtained by Flowers (1980), which was interpreted as a change in rate of visual information processing as the flankers were removed farther into the periphery. In that experiment, there was a two-way interaction between compatibility and SOA for each level of separation, with the maximum difference between RTs to compatible- vs. incompatible-flanker trials peaking later for wide separation. In the present case, however, differences between the RTs for the different flanker conditions remain relatively constant, across SOAs, given the wide-separation condition, except for SOA = 
0 msec. Thus, although the data from Experiment 1 confirm the findings of Flowers (1980) that increased spatial separation changes the time course of processing information from the flankers, the pattern is not as simple as a shift in the time at which a critical processing bottleneck is reached. It is possible that the SOA levels chosen may not have sampled the appropriate flanker lead times. First, would the magnitude of the flanker effect have been greater if a 300-msec interval had been selected? Second, it appears that the increase in spatial separation may have been "overdone," such that the flanker effects were sufficiently attenuated that any interaction with SOA was too weak to be observed. Furthermore, even though the spatial configurations of stimuli were essentially identical to those of Flowers (1980), the reduction in the number of response alternatives may have increased target-processing speed such that target processing no longer overlapped with the processing of flanker elements at that separation (see, e.g., Flowers \& Stoup, 1977).

Notwithstanding these possibilities, which are directly addressed in Experiment 2, the data from Experiment 1 raise the issue that facilitative priming effects may result from at least some processes that differ from those contributing to interference when incompatible flankers are present. If one assumes that the pound sign flanker retains the same degree of "neutrality" across the onset asynchrony levels, the data from the narrow displays suggests that the facilitation effects may follow a time course slightly different from that of the interference effects. Furthermore, the relative elimination of interference, but not of facilitation, with increased spatial separation also suggests possible differences among the underlying causes of the RT changes attributable to flanker-noise compatibility.

\section{EXPERIMENT 2}

Experiment 2 was conducted in order to examine the effects of flanker compatibility over a narrower range of SOA and spacing levels in a digit-naming task otherwise identical to that of Experiment 1. In addition to providing a replication of Experiment 1 using slightly different types of displays, it was assumed that the narrower range of manipulations might reveal a more detailed account of the joint effects of spacing and SOA than the relatively extreme values employed in Experiment 1 did.

\section{Method}

Subjects. Five students and one faculty member at the University of Nebraska served as subjects in a $1-\mathrm{h}$ practice session plus three 1-h experimental sessions conducted on consecutive working days. All subjects reported normal or corrected vision. The five students received $\$ 16.00$ for their participation, and the faculty member (whose summer salary was funded by the NSF support grant) received no additional compensation.

Apparatus. Stimulus generation and response timing were performed by an Apple II computer containing a CCS 7440 programmable timer. The computer was modified so that the video synchronization signal was monitored in one of the "game" inputs, and a voice relay was connected to a second "game" input. Stimuli were displayed on a Sylvania $B / W$ video monitor with a P4 phosphor. As in Experiment 1, the display monitor was located on a table at which the subject was seated. Distance from the subject's eyes to the location on the monitor at which the stimuli appeared was typically about $50 \mathrm{~cm}$, and the visual angle estimates provided below are based on this estimate.

Conditions. Experiment 2 included the same three independent variables as Experiment 1: flanker conditions, SOA, and flanker-target spacing. The three flanker conditions were compatible (e.g., 333), incompatible (e.g., 545), and neutral (e.g., $\left.{ }^{*} 3^{*}\right)$, and were equivalent to Experiment 1 except that the asterisk character replaced the pound sign for the neutral flanker. The digit set 2, 3, 4, 5 served as target alternatives as in Experiment 1. All characters were displayed as normal Apple text characters. The seven levels of SOA were $0,33,67,100$, 133,167 , and $200 \mathrm{msec}$. These were selected to be even multiples of the raster scan period and constituted the delay between flanker and target onset. The three levels of spacing were created by inserting zero, one, or two normal Apple text character spaces between the flankers and the target. Assuming a $50-\mathrm{cm}$ viewing distance, this corresponded to angular distances between the centers of adjacent characters of approximately $1.0 \mathrm{deg}$ per space.

In each experimental session, the subjects were presented with seven blocks of 63 trials each (the first 3 trials of which were "warm-up" and were excluded from analysis). Within each of the three experimental sessions, flanker-target spacing was held constant; each of the six possible orderings of three spacings among three sessions was distributed among the six subjects. Each of the seven blocks of trials run within each session included trials of a different constant SOA level; the ordering of the seven SOA levels within sessions was scrambled by a pseudorandom process. Within each block of trials (following the three warm-up trials for which conditions were randomly selected by computer) there were 12 compatible target-flanker combinations, 12 neutral combinations (asterisk flankers), and 36 incompatible combinations.

The following events occurred on each experimental trial. First, a warning statement (e.g., GET READY FOR TRIAL \#23) appeared for a 3-sec period and was followed by the appearance of a fixation field of three plus signs in the center of the screen, which marked the locations at which the flankers and target were to appear (e.g.,,++++++ , or +++ , depending upon the spacing level). Two seconds later, the flanking plus signs were replaced by the flanker characters, and after a delay corresponding to the SOA, the target digit replaced the center plus sign. Voice RTs were timed from the onset of the target. A typical sequence of events on a given trial, beginning with the fixation field, might be as follows: $+++\rightarrow 3+3 \rightarrow 343 \rightarrow$ (subject says "four"). As in Experiment 1, errors were monitored by the experimenter and edited from the final RT data; however, error rates were again extremely low and were not included in the analysis. The subjects were instructed to respond as rapidly as possible while avoiding errors. The stimulus field disappeared with the response and was replaced by the next "Get Ready" warning after a delay of about $1 \mathrm{sec}$.

\section{Results and Discussion}

As in Experiment 1, overall error rates were too low (less than $3 \%$ ) to warrant analysis by conditions. Mean RT across the six subjects is plotted in Figure 2 as a function of SOA, flanker condition, and spacing. It is apparent that the RTs are substantially faster overall than in Experiment 1; however, this appears to be at tributable largely to the greater visual discriminability of the video character generator of the Apple relative to the system used in Experiment 1. The overall pattern 

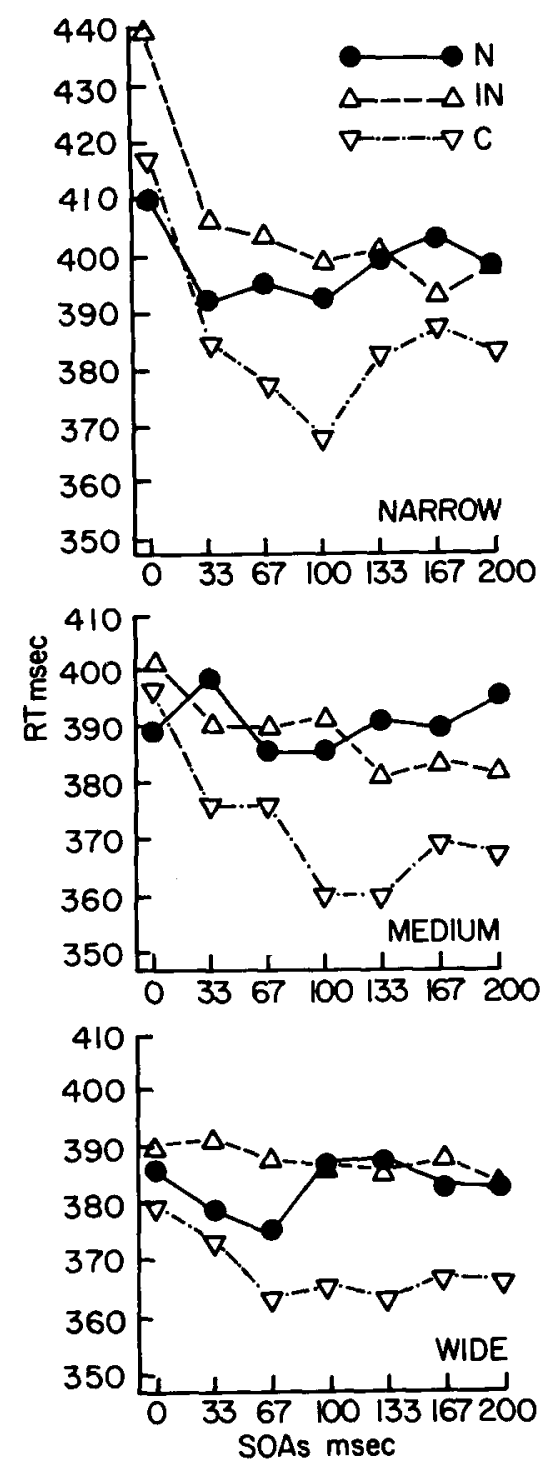

Figure 2. Voice R'Ts from Experiment 2 plotted as a function of SOA and flanker condition (plotted separately for each of the three levels of target-flanker separation).

of results (in particular, the effect of flanker compatibility) and changes in that pattern as a function of spacing bear considerable similarity to what was found in Experiment 1. Significant effects of flanker compatibility were noted at each of the spacings $[F(2,10)=$ $17.3,8.9$, and 10.2 for each spacing level; $p<.01$ in each case]. With the two narrowest spacings, there were also main effects of SOA $[F(6,30)=4.0$ and 3.1 ; $\mathrm{p}<.01$ and .05 , respectively] and SOA $\times$ flanker compatibility interactions $[F(12,60)=3.4$ and $3.7 ; p<.01$ in each case]. Inspection of the graphs for these two spacing levels suggests that the nature of the SOAX flanker condition interaction rests largely upon the changes in the RT differences between the neutral- and compatible-flanker conditions, particularly the comparison of the 0-msec SOA with all other SOA levels. As in
Experiment 1, there is a strong suggestion of some sort of "redundancy loss" or "repetition inferiority effect" that may have competed with the facilitative priming.

A second property of the data obtained with the narrowest spatial separation is that differences between the incongruent condition and the neutral condition (interference effects) are most pronounced at the shortest SOA levels and attenuate at the longer delays. The shape of the function illustrating a monotonic decline in RT to the incongruent target-flanker combinations as a function of SOA in Experiment 2 (with the narrow spacing) is more similar to the shapes of functions found in other experiments (e.g., Taylor, 1977) than to that found in Experiment 1. This finding emphasizes the atypicality of the very small amount of interference noted in Experiment 1 with a 0-msec SOA.

The facilitation effects, on the other hand, appear to build up over the first $100 \mathrm{msec}$, but are maintained throughout the longer SOA levels. Furthermore, the relative amount of interference declines sharply with increases in spatial separation, even though facilitation effects are still noted at the wider separations (also a characteristic of the data obtained in Experiment 1). Taking an appropriate measure of caution in assuming the "true neutrality" of the asterisk flankers, this pattern of data again supports the idea that facilitative priming and response-competition interference are not two manifestations of the same set of process interactions.

It should be noted that with the widest spatial separation, the SOA $\times$ flanker type interaction no longer reached significance and there was no main effect of SOA per se ( $F<1$ for both comparisons). This pattern is thus equivalent to that obtained in Experiment 1 with the side separation of targets and flankers. However, in neither experiment did the widest separation totally eliminate a main effect of flanker type, although the main effect seemed to reside primarily in differences between the compatible and neutral conditions. It thus seems likely that subjects can benefit from compatible visual content lying outside the spatial "beam" within which they are unable to avoid interference from incompatible context.

\section{EXPERIMENT 3}

The third experiment of this study involved a manual (keypress) classification task, rather than the voice reaction time (naming) procedure employed in Experiments 1 and 2. The manual choice RT paradigm is more similar to experimental tasks used by Eriksen and Schultz (1979) and Taylor (1977) and thus adds generality to the pattern of results obtained in Experiments 1 and 2 . In addition, the manual classification task chosen for Experiment 3 is one that mapped several different target alternatives into each of two response categories. This allowed a comparison between the effects of flankers that are physically identical to the target (compa- 
rable to the compatible target-flanker combinations of Experiments 1 and 2) with the effects of flankers that are associated with the same response but are physically different. We have suggested, on the basis of the pattern of data obtained in Experiments 1 and 2, that with the simultaneous presentation of identical flankers and targets, perceptual interference effects may compete with facilitative priming processes, thus obliterating or reversing the facilitative priming effects observed at longer SOAs. Further support for this view would be offered by demonstrating facilitative priming by physically different but response-compatible flankers at the 0 -msec SOA level.

The choice of targets and flanker alternatives used in Experiment 3, which included both letters and digits, also offered an opportunity to assess the relative contributions to RT of compatibility of response-category membership (i.e., whether the flanker and target are associated with the same response key) and semantic category effects (i.e., whether or not the flanker and target are both numbers). LaBerge (1981) and Shaffer and LaBerge (1979) have suggested that responsecategory and semantic-flanker effects can each affect classification time, even when flankers and targets appear simultaneously. Experiment 3 was thus designed to extend those findings through SOA manipulations in order to determine whether semantic-category priming and response-category priming might have noticeably different time courses and whether the two types of priming might be interdependent in some fashion. For example, does facilitative response-category priming depend upon the flanker and target's being from the same semantic category (both letters or both digits)?

\section{Method}

Subjects. Seven undergraduates were each paid $\$ 13.00$ for participation in six experimental sessions of about $\mathbf{4 0} \mathrm{min}$ each. In addition, a practice session of about $30 \mathrm{~min}$ in length was performed on the day preceding the beginning of the experimental session, which allowed each subject to learn the response categories and practice with a sample of trials at each SOA level. The data from one of the seven subjects were not included in the analysis, since a preliminary examination of her data revealed error rates of about .30 , in excess of 10 times the error rate observed from any other subject run in any of the three experiments reported in this study. All subjects reported having normal or corrected vision and speaking English as their native language.

Apparatus. Stimulus display and data acquisition were performed using the same apparatus as in Experiment 2, except that responses consisted of pressing one of two keys on the computer keyboard (the " $F$ " or " $J$ " keys, which were marked with a tape overlay) instead of a voice relay. As in Experiment 2, display timing occurred in even multiples of the raster scan and was initiated with a check of the video synchronization signal.

Conditions. Experiment 3 incorporated the following independent variables. There were two levels of target-flanker spacing (adjacent vs. one character space inserted between the target and flankers), six SOA levels $(0,67,133,200,300$, and $600 \mathrm{msec})$, and six different flanker conditions (as listed in Table 1 and described in detail below). Additionally, there were three replications, or sessions, for each combination of variables, but since only one level of spacing was presented within a single laboratory session, each subject made six separate trips to the laboratory after the practice session.
Table 1

Breakdown of Flanker Conditions in Experiment 3

\begin{tabular}{ccc}
\hline $\begin{array}{c}\text { Label } \\
\text { and } \\
\text { Description }\end{array}$ & Example & $\begin{array}{c}\text { No. of } \\
\text { Instances } \\
\text { Per Block }\end{array}$ \\
\hline & Response* Compatible & \\
ID & DDD & 8 \\
SCSR & NDN & 8 \\
DCSR & 3D3 & 16 \\
& Response Incompatible & \\
SCDR & DRD & 16 \\
DCDR & D4D & 16 \\
& Neutral & \\
N & $* 4 *$ & 8 \\
\hline
\end{tabular}

Note-ID = identical; $S C S R=$ same category, same response; $D C S R=$ different category, same response; $S C D R=$ same category, different response; $D C D R=$ different category, different response. *Classification rule: $D, N, 3,7$ vs. $S, R, 4,5$.

The set of alternative target characters were the Apple text digits $3,4,5$, and 7 and the letters $D, N, S$, and $R$. These characters were mapped into two response categories: $D, N, 3$, and 7 vs. S, R, 4, and 5 . For three of the subjects, D, N, 3, and 7 were associated with a right-hand keypress (the " $J$ " key), while $S, R, 4$, and 5 were associated with a left-hand keypress (the "F" key). The remaining subjects received the opposite responsekey assignment. The flanker alternatives consisted of the set of target alternatives plus the asterisk symbol $\left(^{*}\right)$, which served as a neutral flanker, as in Experiment 2.

Each flanker alternative was paired equally often with each possible target alternative so that response uncertainty was not reduced by attempting to predict the target on the basis of the flanker. However, the different possible combinations of flankers and targets can be broken down into six logical groupings or conditions, as shown in Table 1 . Three of these are responsecompatible conditions, in which the flanker and target belong to the same response mapping, including the identical (ID) condition, same-category/same-response (SCSR) condition, and the different-category/same-response (DCSR) condition. Two conditions were response-incompatible: same-category/differentresponse (SCDR) and different-category/different-response (DCDR). The last condition was the neutral (N), or baseline, condition, in which the flankers were asterisks.

Stimuli were presented in blocks of 75 trials, within which SOA and spacing were held constant. As in Experiments 1 and 2 , the first 3 trials in each block were warm-up trials randomly selected from trials later in the list; data from these first 3 trials were not analyzed. For the 72 trials within each block from which data were collected, the number of trials corresponding to each category of target-flanker condition is shown in Table 1. The lack of numerical balance between conditions is attributable to the constraint that, given a flanker, the conditional probabilities of any target be equal (as in Experiments 1 and 2). Given that blocks corresponding to each combination of spacing and SOA were replicated three times, the total numbers of RT trials contributed by each subject during the experiment for each level of spacing and SOA were 24 for conditions I, SCSR, and $N$ and 48 for the remaining conditions (assuming no errors). For all but the subject excluded from the experiment, errors occurred on fewer than $3 \%$ of the trials and were thus too infrequent to permit a meaningful breakdown by conditions.

On each trial, the sequence of display events was essentially identical to that described in Experiment 2: A "Get Ready" statement, a fixation field $(+++)$, flanker onset $(3+3)$, and, finally, target onset ( $3 \mathrm{~N} 3$ ), to which the subject pressed the appropriate response key. The durations of the "Get Ready" statement and fixation field were identical to those in Experi- 
ment 2 , and the SOA constituted the time between flanker onset and target onset. RT was measured from target onset until key depression.

SOA was held constant within blocks and was ordered within each session by a pseudorandom procedure. Spacing was alternated between sessions, with four subjects receiving the narrow spacing on Day 1 and the remaining subjects receiving the wide spacing on Day 1.

\section{Results and Discussion}

Figure 3 plots the mean RT for each combination of target-flanker relationship and SOA. The top graph plots the data obtained with the narrow displays (no spaces between flanker and target), and the bottom graph plots the data obtained with the wider spacing (one blank space between flankers and target). As the qualitatively similar appearance of the two graphs indi. cates, spacing had little or no overall effect. No significant main effect of spacing was noted, and there were no significant interactions between spacing and any other independent variable. ${ }^{4}$ There were a marginal effect of sessions $[F(2,12)=3.27, .05>p>.10]$ and a marginal interaction between sessions and flanker condition $[F(10,60)=1.98, p<.05]$; however, visual inspection of the data plotted separately for each session suggested that these effects reflected nothing more than the expected practice effects and differential room for improvement with practice among conditions of varying

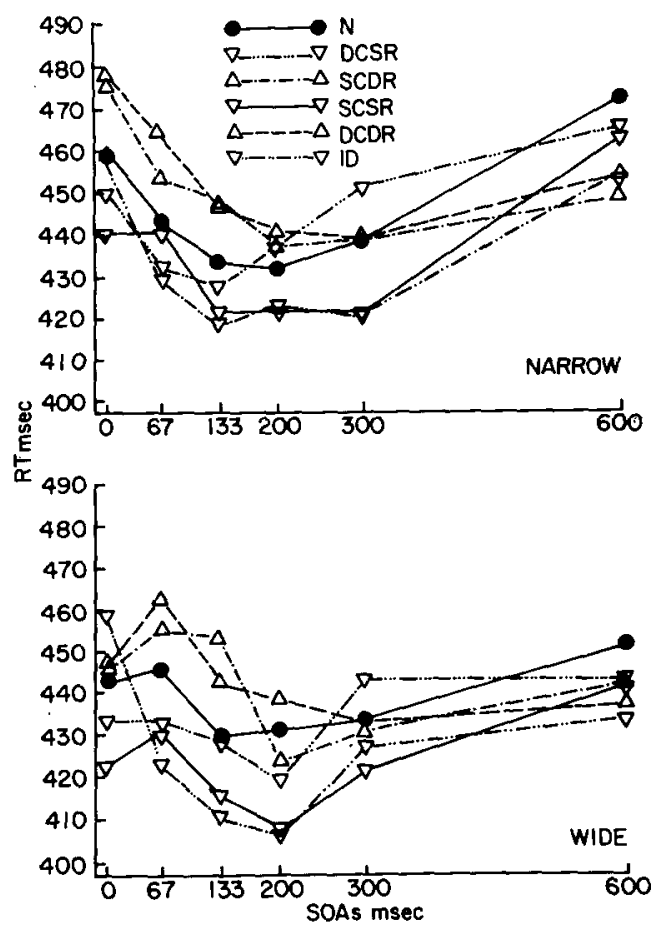

Figure 3. RTs to each flanker condition in Experiment 3 plotted as a function of SOA. The top panel displays data obtained with the narrow target-flanker separation; the bottom panel presents data obtained with the wider separation. difficulty. No ordinal changes or other qualitative differences in the effects of any of the other independent variables occurred over the three sessions.

Of major interest are the effects of flanker condition and changes in those effects over SOA. Both the main effect of flanker condition and its interaction with SOA were statistically significant $[\mathrm{F}(5,30)=5.8, \mathrm{p}<.01$, and $F(25,250)=2.53, p<.01$, respectively] . In general, the pattern of flanker effects obtained with this manual classification task was highly similar to those obtained with voice reaction time in Experiments 1 and 2 . Specifically, interference effects from response-incompatible flankers were present even at 0 -msec SOA (and were maximal with the narrowest spacings). These effects drop off with increasing SOA and are largely attenuated by $200 \mathrm{msec}$ or so.

Facilitation effects (faster RTs to the responsecompatible target-flanker conditions than to the neutral condition) followed a somewhat different pattern across SOA levels. The RTs obtained with the identical condition are very similar to those obtained in Experiments 1 and 2 with the narrow flanker spacings. At 0-msec SOA, the identical conditions produced RTs equal to or slower than those for the neutral condition, with evidence for substantial facilitation at longer SOAs. This facilitation appears to be maintained at longer SOA values than the interference effects obtained with the responsecompatible conditions. However, the response-compatible conditions that did not involve flankers identical to the target, particularly SCSR, produced faster RTs than did the neutral condition, even at the 0-msec SOA level. This pattern of results provides further support for the existence of a "repetition inferiority effect" that appears to be unique to the simultaneous-onset condition and that counteracts potential benefits of response-category priming that appear at longer SOAs. A preplanned comparison of the RTs for the identical and SCSR conditions across the first three SOA levels $(0.133 \mathrm{msec})$ revealed a statistically significant interaction between the two conditions and SOA $[F(2,12)=9.12, p<.01]$. Providing the flankers were not visually identical to the target, benefits were obtained with simultaneous onset.

It should be noted that this pattern of results differs from that obtained by Taylor (1977, Experiment 3), who found little or no facilitation effect when flanking letters that were physically different from the target were presented. In Taylor's study, such "response facilitation" occurred only when subjects were given a strong expectancy bias, that is, when the flanking context actually predicted the response key. Other data (Laberge, 1981; Shaffer \& Laberge, 1979) have, however, provided evidence for facilitative priming by flankers different from the target, particularly when the flankers and target share a common semantic characteristic. We suspect that one key difference between Taylor's (1977) results and those of studies (including the present study) that have shown response-facilitation effects is the complexity of the response mapping. For example, 
Flowers (1974) showed that the use of linguistic context (pronunciation rules and word-nonword distinctions) had little effect on the speeded classification of letter strings when a relatively simple response mapping was used and yet the same form of context produced profound facilitation effects when a slightly more complex response mapping was employed. Both the present study and those of LaBerge (1981) and Shaffer and LaBerge (1979) involved a more complex response assignment rule than that utilized by Taylor (1977).

A final issue addressed by Experiment 3 concerned the distinction between response category-priming effects per se and effects of semantic-category compatibility. For addressing these issues, a comparison among conditions SCSR, DCSR, SCDR, and DCDR was performed. These four conditions were treated as a $2 \times 2$ factorial combination of two levels of response compatibility (whether or not the flankers were associated with the same response key as the target) and two levels of category compatibility (whether or not the flankers and targets were both letters or both digits as opposed to being mixtures of the two types of symbols). Visual inspection of Figure 3 indicates that while category compatibility had little or no effect on RTs to responseincompatible combinations of flankers and targets (i.e., both SCDR and DCDR produced about the same RTs across the different levels of SOA), category compatibility had a rather substantial effect upon RTs to the response-compatible stimuli. Specifically, the facilitation effects of response compatibility seem to be langely dependent upon the flankers and targets' being from the same semantic class of characters-both digits or both letters. Condition DCSR produced RTs that were not substantially different from the neutral condition (about $2 \mathrm{msec}$ faster for the narrow spacing and $4 \mathrm{msec}$ faster for the wider spacing, averaged across SOA levels). In contrast, the ID and SCSR conditions produced facilitation effects averaging $12-14 \mathrm{msec}$ in comparison with the neutral conditions. ANOVA of the $2 \times 2$ combination of response- and category-compatibility levels across SOA confirmed the significance of this pattern. A main effect of both response compatibility and category compatibility occurred [each comparison produced the same $F$ ratio to three decimal places! $-F(1,6)=13.6$, $p<.01$ in each case ]. The interaction between response and category compatibility was also significant $[F(1,6)=$ $7.6, p<.05]$. Despite the suggestion in Figure 3 that the difference in RT between the DCSR condition and the other response-compatible conditions became greater at the longer SOA intervals, the ANOVA reveal neither a significant interaction between SOA and category compatibility $[F(5,30)=1.3]$ nor a significant threeway interaction between response compatibility, category compatibility, and SOA $[\mathrm{F}(5,30)=2.34]$, although the three-way interaction approaches significance $(.15<\mathrm{p}<.10)$. The suggestion that the category compatibility effects may have a slightly different time course than response-compatibility effects per se (and may build up more slowly) is intriguing, despite the marginal evidence obtained in this experiment, and merits further inquiry using different types of stimulus materials.

\section{GENERAL DISCUSSION}

Collectively, these three experiments support the view that $\mathrm{RT}$ in tasks requiring the classification of targets flanked by other information is influenced by the joint effect of a number of different levels of interactions, some of which appear to have different time courses of activation. Modifying the relative time of onset of the flankers and target may serve to change the relative influence of these processes, thereby changing patterns of interference or facilitation of RT caused by the flankers.

\section{Redundancy Losses and Redundancy Gains}

Bjork and Murray (1977), Egeth and Santee (1981), and Estes (1972) have provided examples of tasks in which flanking characters seem to produce a repetition inferiority effect on accuracy of reporting the target, although the generality of their findings has been questioned recently (Eriksen, Morris, Yeh, O'Hara, \& Durst, 1981; Krueger \& Shapiro, 1980). The present data suggest that a repetition inferiority effect may operate in tasks for which stimuli are presented under conditions of high visibility and for which RT is the performance measure. Depending upon the exact form of the stimuli used, the repetition inferiority effect may not necessarily lead to slower RTs than those produced by neutral noise or flankerless control conditions, but it may serve to cancel out the potential benefits of facilitative priming effects that are observed when the flankers lead the target in time (Experiments 1 and 2) or that occur with the simultaneous onset of flankers and targets that differ physically but belong to the same response category (Experiment 3 ). We would argue, therefore, that although the disruptive effects of character repetition may be very subtle and thus easily ob: scured by other processes occurring in a target recognition or classification task, such effects probably do exist.

\section{Facilitation vs. Interference}

The flanker compatibility effects observed in all three experiments suggest that interference effects caused by incompatible flankers and facilitation effects attributable to compatible flankers cannot be interpreted simply as two manifestations of a single response-priming process. First, the interference effects (operationally defined as greater RTs to the response-incompatible flanker trials than to the "neutral" trials) occur langely at the shorter SOA levels, whereas the facilitation effects (faster RTs to the compatible flanker trials) seem to be maintained at longer SOA intervals. Second, increasing flanker-target spacing (except in Experiment 3, in which the spatial manipulation appears to have been too slight 
to cause a substantive effect of any sort) tended to attenuate the interference effects while maintaining facilitation. Keeping in mind that the quantification of facilitation and interference depends upon the arbitrary assumptions that the pound sign or asterisk neutral flanker is an appropriate baseline and that its neutrality is not affected by SOA, the present results suggest that human observers may effectively block out disruptive information under conditions in which benefit can still be obtained from information that is compatible with the response to the target.

\section{Semantic Category Effects}

The finding in Experiment 3 that semantic-category compatibility (whether or not the flankers and target contained a mixture of letters and numbers) occurred only for the response-compatible flankers provides additional evidence that facilitative priming effects involve different processing levels. This pattern suggests that response competition may result from a very rapid involuntary activation process (or set of processes) that have largely died away by the time the outputs of other processes (e.g., semantic categorization) are available. Perhaps the slower, "more detailed" activation processes do not produce a sufficiently high level of activation to exceed a decision criterion for the "wrong" response (hence, response competition is avoided) and yet still contribute to the level of activation for the correct response when the target provides confirming information.

Additional empirical evidence on the time course of category-priming vs. response-compatibility effects is needed before a useful process model that describes these cognitive activities can be outlined in detail. However, the present data and those of other recent experiments do clearly suggest that response activation is influenced by the joint contribution of several qualitatively different forms of stimulus evaluation having overlapping time courses. It is thus increasingly evident that stimulus "encoding," "cognitive" processing, and response activation cannot be viewed as discrete and isolatable stages.

\section{REFERENCE NOTES}

1. Santee, J., \& Egeth, H. Feature-specific inhibition vs. perceptual independence in letter identification. Paper presented at the annual meeting of the Psychonomic Society, Philadelphia, November 1981.

2. Flowers, J. H. Priming effects of noise elements in visual displays. Paper presented at the annual meeting of the Psychonomic Society, St. Louis, November 1980.

\section{REFERENCES}

Bjork, E. L., \& Murray, J. T. On the nature of input channels in visual processing. Psychological Review, 1977, 84, 472484.

Duncan-Johnson, C. C., \& Kopell, B. S. The Stroop effect: Brain potentials localize the source of interference. Science, $1981,214,938-940$.
Egeth, H. E., \& Santee, J. L. Conceptual and perceptual components of inter-letter inhibition. Journal of Experimental Psychology: Human Perception and Performance, 1981, 7, 506-517.

Eriksen, B. A., \& Eriksen, C. W. Effects of noise letters upon the identification of a target letter in a nonsearch task. Perception \& Psychophysics, 1974, 16, 143-149.

Eriksen, C. W., \& Hoffman, J. E. The extent of processing of noise elements during selective encoding from visual displays. Perception \& Psychophysics, 1973, 14, 155-160.

Eriksen, C. W., Morris, N., Yeh, Y., O'Hara, W., \& Durst, R. T. Is recognition accuracy really impaired when the target is repeated in the display? Perception \& Psychophysics, 1981, 30, 375-385.

ERIKSEN, C. W., \& SchulTz, D. M. Information processing in visual search: A continuous flow conception and experimental results. Perception \& Psychophysics, 1979, 25, 249-263.

Estes, W. K. Interactions of signal and background variables in visual processing. Perception \& Psychophysics, 1972, 12, 278286.

Flowers, J. H. The effect of correlated linguistic dimensions on speeded classification of visually presented trigrams. Memory \& Cognition, 1974, 2, 372-378.

Flowers, J. H. Response priming effects in a digit naming task as a function of target-noise separation. Bulletin of the Psychonomic Society, 1980, 16, 443-446.

Flowers, J. H., \& SToup, C. M. Selective attention between words and colors in speeded classification and vocalization tasks. Memory \& Cognition, 1977, 5, 299-307.

Hock, H. W., \& EaETH, H. E. Verbal interference with encoding in a perceptual classification task. Journal of Experimental Psychology, 1970, 83, 299-303.

Knueger, L. E., \& Shapiro, R. G. Repeating the target neither speeds nor slows its detection: Evidence for independent channels in letter processing. Perception \& Psychophysics, 1980, 28, 68-76.

LABEROE, D. Unitization and automaticity in perception. In J. H. Flowers (Ed.), 1980 Nebraska Symposium on Motivation: Cognitive processes (Vol. 28). Lincoln: University of Nebraska Press, 1981.

MCClelland, J. L. On the time relations of mental processes: An examination of systems of processes in cascade. Psychological Review, 1979, 86, 287-330.

Miller, J. Discrete versus continuous stage models of human information processing: In search of partial output. Journal of Experimental Psychology: Human Perception and Performance, 1982, 8, 273-276.

NeELY, J. H. Semantic priming and retrieval from lexical memory: Roles of inhibitionless spreading activation and limited capacity attention. Journal of Experimental Psychology: General, 1977, 106, 216-254.

Schvaneveldt, R., \& Meyen, D. E. Retrieval and comparison procedures in semantic memory. In S. Kornblum (Ed.), Attention and performance IV. New York: Academic Press, 1973.

Shaffer, W. O., \& LaBerge, D. Automatic semantic processing of unattended words. Journal of Verbal Learning and Verbal Behavior, 1979, 18, 413-426.

TAYLOR, D. A. Time course of context effects. Journal of Experimental Psychology: General, 1977, 106, 404-426.

\section{NOTES}

1. Recently, Eriksen, Morris, Yeh, O'Hara, and Durst (1981) failed to find evidence for tepeated letter inferiority effects, even under conditions of brief exposure duration and using accuracy as the dependent variable. These authors have suggested that the effects reported by Bjork and Murray (1977) and Egeth and Santee (1981) may be critically dependent on masking phenomena, as well as subject to some subtle sources of response bias imposed by the design.

2. We attribute the slightly higher error rate reported by 
Taylor (1977) to the fact that SOA was not blocked in his study as it was in ours. The rates we obtained were far too low to warrant a breakdown by conditions. However, in both this experiment and the others reported in this article, higher error rates were associated with longer response latencies, and thus there was no evidence of a speed-accuracy tradeoff.

3. On the basis of comparing data from the present experiments and data from previous published and unpublished experiments obtained using the Tektronix monitor and the Applecontrolled video screen, we strongly suspect that relative discriminability of the characters in the two types of displays may play a role in determining the SOA value producing maximum interference. For experiments in which we have used the Tektronix monitor, which involve larger and more diffuse dot matrix characters than the more "normal"-appearing text generated by the Apple, maximum RTs to incompatible flanker-target stimuli with the narrow spacing have often occurred, not at 0 -msec SOA, but at some small positive SOA value (e.g., $50-100 \mathrm{msec}$ ). With the more compact Apple text characters (which have produced considerably shorter response latencies in general), the closest spacing between targets and flankers has almost universally led to the greater interference effects at 0 -msec SOA.
Since the Tektronix characters are less discriminable and extend farther into the periphery, the speed of processing the flankers relative to that of processing the target may indeed be slower than with the Apple text characters or with printed text in a standard tachistoscopic display, which therefore changes the function relating interference to SOA. Note that a similar change in this function occurs with the Apple displays as spatial separation is increased. Essentially, the "narrow" displays on the Tektronix scope are effectively less "narrow" than those produced with the Apple.

4. Comparison of the RTs obtained with the two separation levels does show some of the trends as observed in Experiments 1 and 2-a greater emphasis on facilitation vs. interference with the wider displays and a suggestion that the maximum flanker effects may occur at somewhat longer SOAs (particularly with the interference effects). We suspect that, had we used a wider separation, some of the same type of interactions we obtained between separation and other variables in Experiments 1 and 2 might also have been obtained here.

(Manuscript received January 29, 1982; revision accepted for publication August 26, 1982.) 